\title{
Robust Action Selection by the Robot in Human-Robot Interaction (HRI) Environment
}

\author{
Kamran Ashraf \\ Department of Computer Science \\ National Textile University \\ Faisalabad, Pakistan
}

\author{
Muhammad Asif, PhD \\ Department of Computer Science \\ National Textile University \\ Faisalabad, Pakistan
}

\begin{abstract}
With the evolution in the auspicious field of Human Robot Interaction it is crucial to work for the Robust Action Selection on a robot's end especially when the human exhibits an unknown behaviour. A particular human action may lead to more than one human behaviour(s) and when it comes to a robot as an assistant or a co-worker it is of vital concern to have some efficient method to select a suitable action performed by the robot in response. After exploring multiple techniques a novel method is suggested using RL based approach to cater the need of robust action selection with addition of domain knowledge. Experimentation is performed using hardware equipment including 4DOF Robotic Arm equipped with the Arduino Kit and 480x640 Camera. Very promising results have been found and future direction is discovered.
\end{abstract}

\section{Keywords}

Robotics, Human Robot Interaction, Service Robots, Reinforcement Learning

\section{INTRODUCTION}

Today millions of robots are serving mankind in a variety of ways but still in a lay man's view they are thought of as something that should remain within a confined environment. This type of stereotype associated with the robots is changing on the same rapid pace as the pace of progress in the vibrant field of Human Robot Interaction (HRI), a promising zone under the umbrella of Human-Computer Interaction (HCI).

Human-Robot Collaboration (HRC) is gaining interest in wide range of applications such like manufacturing [1] and private services [2]. Human Robot Interaction (HRI) mainly deals with the robot behavior learning according to the action of interacting humans [3]. There are many different approaches presented in the literature for human behaviour recognition and robot behaviour selection in response but these approaches suffer from different limitations.

One of the main challenges in the field of HRI is the intuitive action selection by a robot in response to both known and unknown human behaviours in order to provide a seemingly natural communication environment to minimize hesitation gestures commonly posed by the human in HRI applications. A major reason of these hesitation gestures is the slow and paused response of the robot that makes a sense of dealing with something mechanical even in case of humanoid robots, those are supposed to act as good companions, assistants and co-workers in multiple settings [4].

There is an acute need of suggesting novel approach(es) in order to achieve HRI goals by thoroughly finding the limitations presented by the work done in this field to-date.

This study is dedicated to the problem of robust action selection by a robot in response to human behaviour especially when the action sequence is unknown and it is contributing by developing/improving the algorithm(s) to address this problem. The study also sets path for the potential young researchers in this area and invites research community to contribute to this multidisciplinary and challenging field of HRI by addressing other associative questions/problems. A very stable approach of RL is used for action selection in unknown scenarios with the addition of the domain knowledge.

With the domain knowledge in hand it can be made less complex for a robot to select a suitable action in response to the unknown human behavior. It is further assumed that during HRI a human performs only current domain related action(s), it means every action performed by the human will be analyzed by the robot in the context of the current domain. Robot's action will be selected given the domain knowledge (knowledge of HRI workspace) and the human action with the condition of efficiently selecting the action.

\section{REVIEW AND LITERATURE}

In this section related approaches and the difference of proposed and other approaches is discussed. RL is an interesting and researched upon topic. There are many solutions available in the literature of RL human input is not required. Here the related work done using different approaches is discussed.

There can be a lot of trials required to program by supervisory information. Learning the solution of problems with demonstration is used in [5]. Programming by Demonstration is different field in this scenerio. In $\mathrm{PbD}$ a robot watches a demonstration and learns the tasks to be performed. PbD offers a lot of such solutions which deviate from main focus of HRI. The approaches discussed in [6] applies RL to the field of $\mathrm{PbD}$.

Approaches dealing with premature human intentions is described in [7]. These approaches [7] represents method based on probability which helps the robot to act according to human behavior but the described approach is not useful when intentions are not already modelled.

\subsection{Exploration of the Probabilistic Models}

The DBN, HDBN and HMM are the techniques those require a large number of post and pre conditions and are really complex techniques to implement. The complexity obviously increases the levels associated with the overhead. Complexity reduction is the main issue to deal with these techniques. Keeping all these shortcomings in mind a solution is presented that deals with the mentioned problems:

- A Bayesian Network Model: One of the most effective classifier is the Bayesian classifier. A Bayesian network is a directed graph that is marked 
and also directed. It encodes probability of arbitrary variables [8].

- Hidden Markov Model: A model based on statistical Markov is HMM. In hidden Markov model hidden states are used and assumption is made about the system to be Markov process. These models are mainly used in the field of pattern recognition, gesture detection and other same type of scenarios, discharges which are partial. A study [9] uses HMM to recognize the human intention by discussing in detail the usage of HMM for modelling the human intention. In the technique of HMM, each observation corresponds to a hidden state and in case of intention recognition if the human performs more than one action corresponding to the same intention then all the hidden states must point to the uniform human intention. HMM is good for speech recognition where each phonic corresponds to a distinct phonogram.

- Particle Filter Method: Particle filters are said to be methods for solve the filtering problems, there are also called Sequential Monte Carlo (SMC). In probability scenarios these are recognized as mean field particle interpretations of Feynman-Kac probability measures [10]. Particle filters have been found very promising in estimation problems [11]. In the past particle filters were used in estimation problems of robot localization but now the researches have applied it to robotic domain with very useful effects [12].

\section{MATERIALS AND METHODS}

Here it is supposed to perform the process of Selection of the Suitable Probabilistic Model for efficient action selection. As the problem falls in the category of the linear problems, probabilistic model(s) suit it well. PF through RL is sued.

The topic of spontaneous HRI is not very much explored in the situations where human intentions are unknown. The approach proposed describes a situation where human intention is unknown but the HRI scenario is known to the robot. The robot system is aware of the objects preset in the workspace, it is also aware of actions that a human intends and the modifications that can happen in the scene but the robot is unaware of human intention which means that it does not know what human intends to do in the scene. Further the robot has the domain knowledge which reduces the complexity as the robot is already aware of the workplace and the probable actions that can be performed in that workplace.

In scenarios related to unknown human intention the following properties are considered [13] to intuitively interact with human:

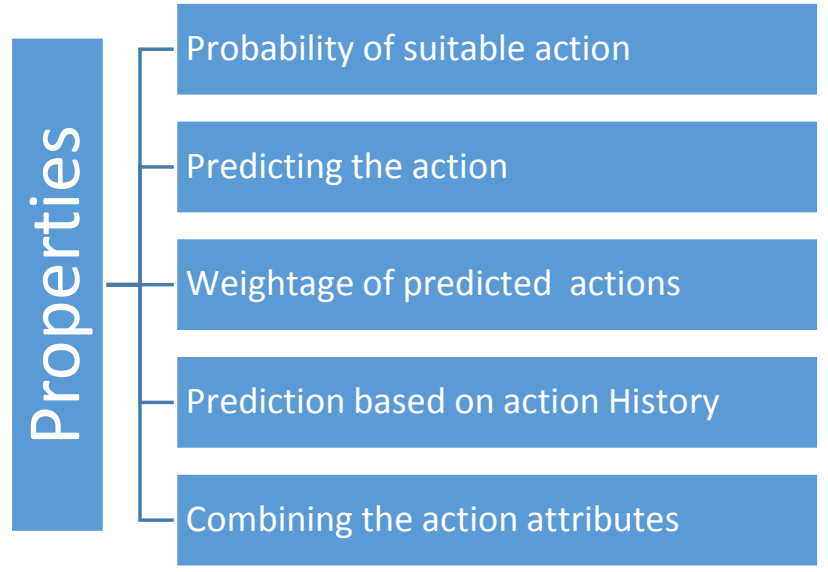

Fig. 1: Properties for intuitive interaction [13]

\section{EXPERIMENTS}

Experimentation is performed using hardware equipment including 4DOF Robotic Arm equipped with the Arduino Kit and $640 \times 480$ Camera. The workspace is used to interact between human and robot. The camera used to capture video data with $640 \times 480$ pixel frame size and 25 frames per second frame rate. $\mathrm{C}++$ has been used as the programming language to implement HRI and analyze the image. The robot communication is done through Arduino Kit and the reactions are understood by $\mathrm{V}++$ programming language. With human performing actions there are changes in scene which are supposed to be human actions. Two main features characterizes the actions during the experimentation.

- Distance between the objects

- $\quad$ Placement of the objects

Objects used in experimentation are blocks and placed on the table. Human intention based placement of objects is done during experiments. Tasks represent human intentions and these intentions are defined by trajectories. A trajectory is represented in a plane in the two dimensional scenario. Distance of objects and positioning is given as axes. A point shows action. Angle is calculated for the drop of line occurring through instant points.

In experiment reactions are selected, now at first the reason of selection and also the structure is discussed. After that the experimentation results are explained. The selection of reaction is based on a method which is given here. The human intentions which are unknown and needs to address in this case corresponds to composition of objects.

Objects are arranged in vertical shape with respect to unknown human intentions. Blocks are placed in a random fashion on starting the experimentation. The human then selects and positions the block at a specific point on table. This point is shown in Figure 1 at $(310,125)$. After this placement the human once again places a second block vertically near the previous block as shown in Figure 1 at point $(310,170)$ by block. It is assumed that $\theta_{-} 1$ and $\mathrm{d}_{-} 1$ lare the distance and angle of these two blocks positioned by human. On the basis of action observation a hypothesis is formed. This hypothesis is shown in Figure 1 with the green circle and it is considered to be the probable place on which block will be positioned next. 


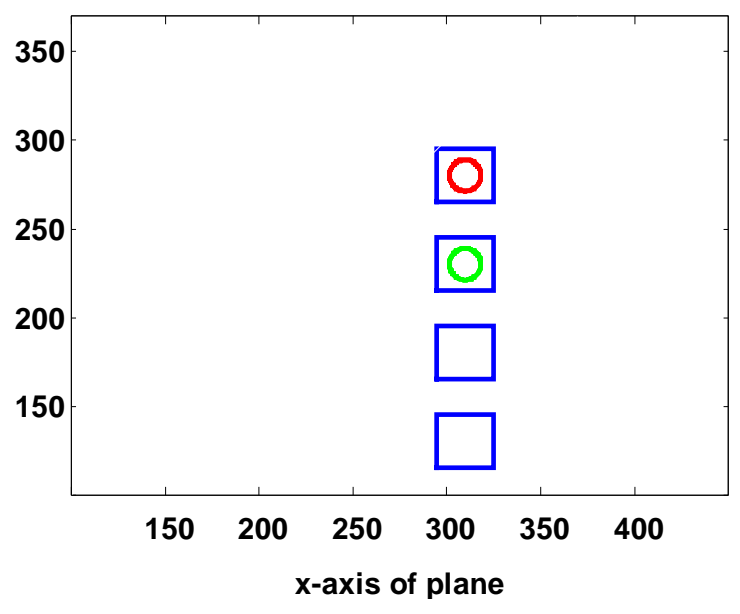

Fig. 2: Graph showing hypotheses of intentions

On occurrence of first reaction the past history and conditional probability is not available so in that case previous probability of the action performed in exercised. Predicted actions get uniform weight if there is no past history available for the action series during HRI. Then only highest valued reactions are present for the system. An example is taken where blocks are placed vertically by $\theta$ angle and distance $d$. Purple cross shows the weightage of hypothesis on the very first interaction step shown in Fig. 2. In case human terminates robot action and send the correction then the system is updated by potential actions and adds the action corrected by human to the table and then updates the records in previous and conditional probability table. The corrected action is also updated in the series of HRI actions. On acceptance of the reaction the system only updates the table but no need to add new action to the table. The robot creates another hypothesis shown in Fig. 2 by red circle. As robot has only witnessed one action that's why only one hypothesis is created for the following place in vertical space.

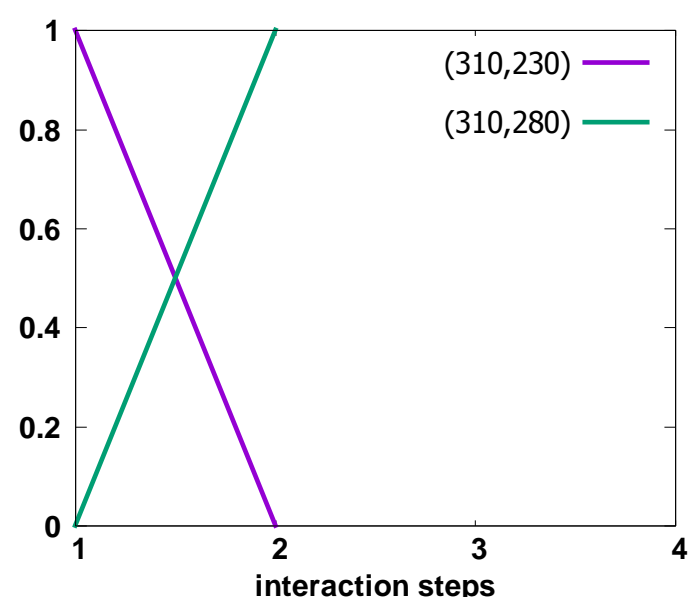

Fig. 3: Graph showing weightage with respect to intentions

Human accepts the reaction of robot because the intention of human is also the same which is to position the blocks vertically. The series of actions is stored by the system after the completion of each interaction. Human action, correction and reaction of robot are the part of stored series.

On acceptance of any hypothesis it is removed and its value becomes zero as presented in Fig. 3, the second interaction step shows it as green line moves to zero.

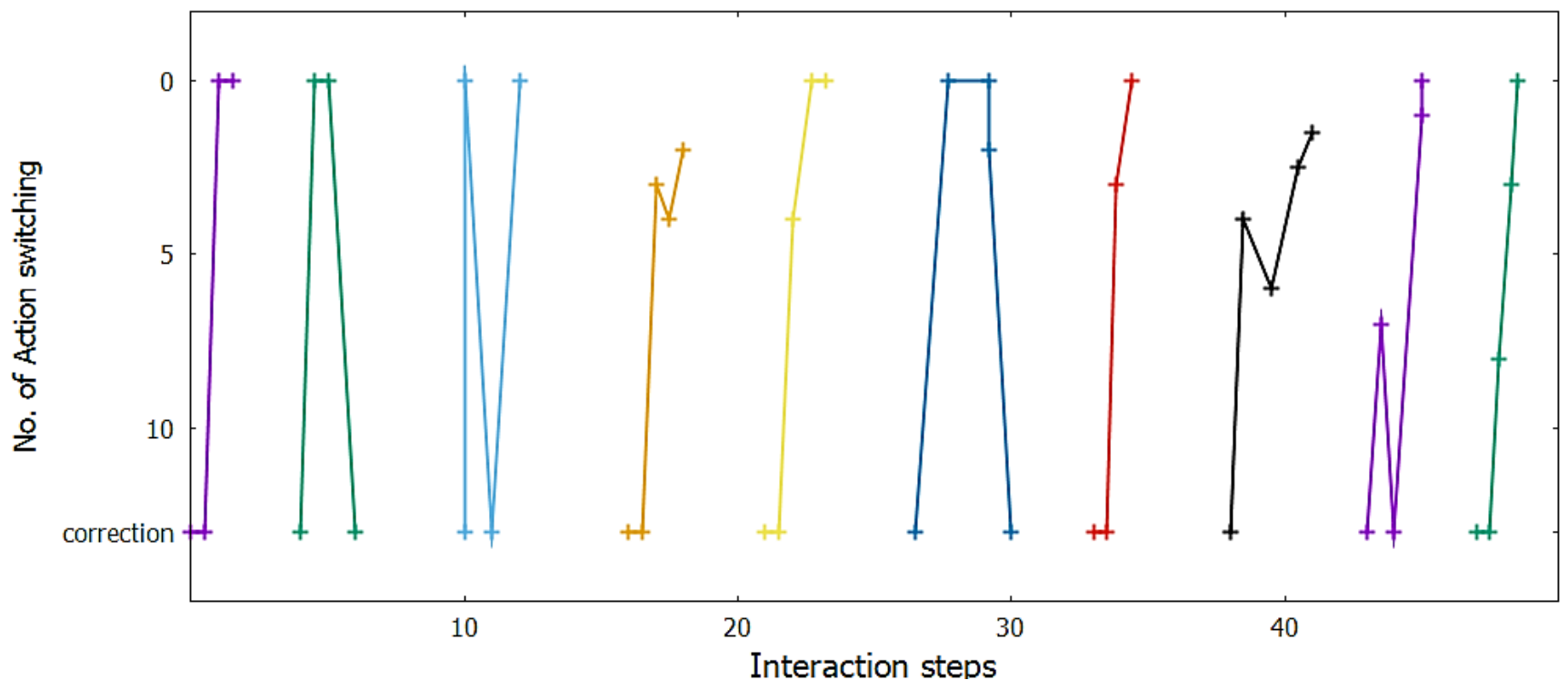

Fig. 4: Robot reaction graph, intention is unknown

Fig. 4 shows a graph of interaction tasks without domain knowledge. The results are formulated by 10 different tasks independently. The horizontal axis of the graph is used to represent interaction steps whereas vertical axis is showing the number of actions switched. This switching is done on the request of human during interaction with the robot. The following results shows that $69 \%$ reactions were acceptable which means that human accepted those reactions and robot system predicted it correctly according to human intention. The remaining $31 \%$ of reactions were not accepted and the human corrected the actions. After correction it is found that nearly half of the reactions out of $31 \%$ got accepted on 2 nd interaction step. Switching rate is also found to be good as $40 \%$ of the accepted reactions had maximum three times switching of actions, $25 \%$ reactions had maximum five actions switched and only $8 \%$ had switching of seven actions. 


\section{CONCLUSION}

Probabilistic methods are discussed to select the reaction of a robot when intention of human is unknown. In this approach the robot is unaware of human intentions so the robot makes an estimated reaction during the HRI. In case the robot action is according to the intention of human then it gets accepted.

These experiments can be applied to different domains where cooperation is required. There can be other actions associated in the scenarios, the presented pick and place method is only one of them. An example of a human working in textile sector domain can be taken. Here the human performs actions like knitting, cutting, packing the gloves and the robot is required to help him in his work by handing over the required items to the human. Whenever the human is going to knit the glove the robot makes prediction for the human intention and provide the required gloves for knitting purpose from the previous department. In this method the predicted actions depend on the human intentions, as the human tries to work on something the robot makes prediction and on robotic reaction the human accepts or corrects the actions. This procedure can be applied in other complex fields as well. It's already discussed that complexity reduction is the main thing in this scenario and when the robot is unaware of the objects used and human intention, the action selection is complex in unknown human intention scenario. The actions selection becomes robust in this case by weighting the action hypothesis. If the action prediction is complex for the robot then the weight of hypothesis is difficult to assign to different tasks. With the addition of domain knowledge the robot is already aware of the workplace and this can make it easier to choose the reactions of robot. Domain knowledge can also be used to get better results for prediction of action and the future work can be done on this aspect.

\section{REFERENCES}

[1] R. Wilcox, S. Nikolaidis, and J. Shah, "Optimization of temporal dynamics for adaptive human-robot interaction in assembly manufacturing," in Robotics: Science and Systems, 2012.

[2] H. Goto, J. Miura, and J. Sugiyama, "Human-Robot Collaborative Assembly by On-line Human Action Recognition Based on an FSM Task Model," in HumanRobot Interaction 2013 Workshop on Collaborative Manipulation, 2013.
[3] Goodrich M. A and A. C Schultz, "Human-robot interaction: a survey," Foundations and trends in humancomputer interaction, vol. 1, no. 3, pp. 203-275, 2007.

[4] R. O. Ambrose et al., "Robonaut: NASA's space humanoid," IEEE Intelligent Systems and Their Applications, vol. 15, no. 4, pp. 57-63, 2000

[5] M. Kaiser and R. Dillmann, "Building elementary robot skills from human," In Proceedings of the IEEE International Conference on Robotics and Automation, pp. 2700-2705, 1996.

[6] Rosenstein. M and Barto. A., "Supervised actor-critic reinforcement learning," Handbook of learning and approximate dynamic programming, p. 359, 2004.

[7] Schmid. A, Weede. O, and Worn. H, "Proactive Robot Task Selection Given a Human Intention Estimate," Robot and Human interactive Communication, ROMAN. 16th IEEE International Symposium, pp. 726-731.

[8] Friedman, Nir, Dan Geiger, and Moises Goldszmidt, "Bayesian network classifiers," Machine learning, vol. 29, no. 2-3, pp. 131-163, 1997.

[9] Hofmann, A. G., and B. C. Williams, "Intent Recognition for Human-Robot Interaction," Interaction Challenges for Intelligent Assistants, 2007.

[10] Del Moral and Pierre., "Feynman-Kac formulae," Genealogical and interacting particle approximations, 2004.

[11] Nummiaro, Katja, Esther Koller-Meier, and Luc Van Gool, "An adaptive color-based particle filter," An adaptive color-based particle filter, vol. 21 , no. 1 , pp. 99 $110,2003$.

[12] Thrun and Sebastian., "Particle filters in robotics," In Proceedings of the Eighteenth conference on Uncertainty in artificial intelligence, pp. 511-518, 2002.

[13] Awais M. and Henrich D., "Human-Robot Interaction in an Unknown Human Intention Scenario," In Frontiers of Information Technology (FIT), 2013 11th International Conference on. IEEE, vol. 89-94, 2013 